\title{
Wildlife Deterrence from Hazards Using High Brightness Ultraviolet Light
}

\author{
Donald J. Ronning* \\ Lite Enterprises Inc, 4 Bud Way, Ste. 15, Nashua, NH 03063
}

\begin{abstract}
The purpose of this study was to examine the behavioral impact of varying LED (light emitting diode) spectral emissions on avian species. A novel oculo-neuro-motor response to monochromatic LEDs has been identified. LED colored light of sufficient intensity matched to the short-wavelength cones of avian species can efficiently diminish overall visual perception leading to an augmented behavioral response. The study method involved monitoring the change in behavioral response after exposure to high brightness monochromatic LED light to wild Osprey (Pandion haliaetus) foraging and nesting in their natural environment. Our results demonstrated a statistically significant change in foraging behaviors with wild Osprey $(\mathrm{p}=0.005)$ and in nesting behavior as a result of exposure to high brightness monochromatic LED light. An augmented behavioral responses was observed which is believed to be analogous to human reaction to solar glare and glint. The neuroimaging mechanism of avian species is discussed. This novel augmented behavioral response provides a new technique of studying the neuroscience of temporal and spatial light stimulus. A non-lethal deterrence technique involving the augmented behavioral response could mitigate mortality and morbidity of avian-human conflict in areas such as airports, airplane bird strikes, and wind turbines.
\end{abstract}

Key words: Avian vision, ultraviolet LED, non-lethal deterrence, solar glare, solar glint.

\section{Introduction}

Human wildlife conflicts can be managed through a variety of techniques. Current mitigation strategies have limited effectiveness and are prone to habituation with repeated long-term exposure. The recently developed technology of LEDs (light emitting diodes) and the discovery of avian oculo-neuro-motor response to monochromatic light offer a novel mechanism for non-lethal mitigation of these conflicts.

Gene sequencing has identified that all vertebrate visual photo pigments opsin genes belong to five gene families. The five gene families consist of one rod and four cone pigments with peak spectral absorption within the following ranges: rod pigment (Rh1, 450-540 nm) and four cone pigments (SWS1, 350-450 $\mathrm{nm}$ ), (SWS2, 400-470 nm), (Rh2, 460-530 nm), and (LWS, 480-570 nm) which are represented in various birds, fishes and reptiles [1]. It is believed that specie

*Corresponding author: Donald J. Ronning, B. S. chemistry, B. S. photographic science, research field: avian vision and behavior. evolution and adaptation has driven gene-sequence variation within each of the photo pigments families which can be tuned to absorb preferentially across a restricted range of the spectrum [2]. Two variants of ultraviolet color vision have been identified in birds with a pronounced difference in the wavelength of peak absorbance of the SWS1 cone. The UVS variant involves peak absorption SWS1 (360-373 nm) while the VS variant involves peak absorption SWS1 (402-426 nm). The VS variant is the most common and has the SWS2 (430-463 nm) peak absorption slightly shifted towards longer wavelengths [3]. The literature also identifies the cones pigments by alternative names: SWS1 (UVS or UV), SWS2 (Blue or Violet), Rh2 (M or Green), and LWS (L or Red). Several avian species have a unique class of double cones which may comprise more than $50 \%$ of the cones whose function is not well understood [4]. The spectral range of the cones often have overlapping sensitivities. Some avian species have a dominant percentage of double cones consisting of Green and 
Red cones which exhibit variations of the cone taper and size which are known to be neurologically linked. Double cones are believed to play a significant role in the detection of object edges and motion.

Even though mammal and avian species followed different evolutionary paths that resulted in differences in sensory systems and brain structures, they share many common genetic, biological, and neurological similarities to humans. The purpose of UV vision for avian species includes orientation, foraging and signaling [5]. UV vision in avian species has been of great interest as it gives birds an additional dimension in the color space, which is lacking in most mammals [6]. Differences in contrast sensitivity, spatial frequency sensitivity, and rod/cone concentration between species are readily attributed to basic behaviors observed for a particular specie within its environment (e.g. diurnal, nocturnal). Studies have identified the significant role that UV vision has in avian mate selection and foraging [7]. Spectral sensitivity and color vision in birds have been studied more extensively than temporal resolution and luminance contrast [8].

Studies involving measurements of the light level difference that an observer needs to discriminate a light source as flickering versus steady intensity under varying temporal and spatial conditions provide insight to the contrast sensitivity (dynamic range) of the species visual system. Temporal spatial contrast sensitivity not only varies from specie to specie, but within species under scotopic (dark adapted) and photopic (light adapted) conditions. Pupil dilation is the dominant mechanism for the eye to adapt changing conditions of illumination. Increasing light intensity improves the flicker detection [9]. The ability to perceive movement varies with different temporal and spatial variations as well as wavelengths of light [10]. The ability to visually perceive movement involves the biological components of the eye as well as the neurological mechanisms of the optic nerve and brain functions. Object recognition requires synaptic nerve activity associated with higher levels of brain function leading to behavioral responses which may be voluntary or involuntary.

The eye structure, pupil size, number and distribution of cones vary from specie to specie but the biological and neurological processes are similar. Neuro wiring between the photoreceptors and the optic nerve follows a basic circuit design processing sequence-temporally filtered signals from individual receptors, rectified by a threshold mechanism, then summed (positively or negatively) over many receptors. In humans, the contribution of short-wavelength-sensitive (Blue) cones to the neurological signals from the eye is disproportionately higher than from Green and Red cones [11]. The avian mid-brain visual functions and isthmotectal circuitry, which control the ascending flow of retinal signals carried to higher visual areas of the brain, are similarly organized to vertebrate class species [12]. Exceeding the dynamic range of any of the cone channels results in a saturated neuro impulse signal in which no or very limited information can be derived.

Behavioral responses resulting from a condition of an overwhelmed neurological impulse initiated from a flash of bright light effectively interferes with visual perception. Discomfort glare is called an instinctive desire to look away or it creates difficulty in seeing a task. Disability glare impairs the vision of objects arises by the inter-reflection of light within the eyeball resulting in reduced contrast. Dazzle describes the situation when intense glare completely impairs the vision. A broad spectrum of white light or sunlight is commonly associated with these types of visual impairments. Our study utilized a narrow spectrum of light which overwhelms a single channel (SWS1 cone) of the avian visual system to induce the augmented behavioral response to effectively block the perception of objects. The observed behaviors may be similar to either discomfort glare, disability glare, or dazzle but the neurological mechanism are different. Neither image acuity, chromatic contrast, discomfort glare, 
disability glare, nor dazzle mechanisms are involved. Intensities less than those required to overwhelm the visual perception of the species, remain effective in alerting the specie to a changed environment resulting in increased awareness and attention to the light source.

The mechanism of augmented behavioral response involves the stimulation of short-wavelength-sensitive, UV or UVS cones, to exceed the dynamic range of a light adapted eye of a dominant neurological color channel involved in edge detection, motion detection, and other differentially processed neurological signals that are transmitted from the ganglia to the brain via the optic nerve. Our prior field activities had identified that the strongest behavioral responses for a given light intensity resulted from spectrum emissions that were closely matched to the short wavelength cones of the avian species. The peak absorption of the Osprey SWS1 cones is $405 \mathrm{~nm}$ [13].

Sudden oculo stimulation of this type can be reinforced with other sensory stimuli. The use of ultraviolet or deep blue colored LEDs has the benefit of minimizing any distraction to humans. The non-lethal nature of using high brightness LEDs as a wildlife deterrence producing altered animal behaviors of increased awareness and avoidance can be beneficial in mitigating mortality, morbidity, and economic loss in a wide range of human-wildlife conflict such as aircraft wildlife strikes, predation loss and selective harvesting at agricultural and aquaculture production sites.

The functional role that the SWS1 cone plays in avian visual perception is not well understood. The role that artificially high intensities of illumination that exceed the dynamic range of the SWS1 cones in relationship to the other color channels has not been previously studied. Given the different color opponent mechanisms which are known to be present in human vision, it was postulated that similar mechanisms exist in avian species. The retinal model for avian species predicts degraded visual perception from a flickering high intensity light that is well matched to the SWS1 cone peak absorption spectrum. The combination of additional monochromatic LED light to a scene would result in an unbalanced, unnatural chromatic color distribution and the pulsating brightness will cause a condition that the avian eye is unable to accommodate normal visual perception due to the unbalanced optical flow being delivered to the brain. Therefore, the authors predict that avian species would exhibit changes to their behavioral patterns.

The purpose of this study was to induce a behavioral response of sufficient strength to alter the foraging and nesting behaviors of wild Osprey in their natural setting through the use of the spectral properties of light to affect the temporal resolution of the avian visual system by utilizing a pulsing monochromatic LED source capable of narrow spectrum emission.

\section{Experimental Section}

\subsection{Experimental Methods}

Two separate studies were conducted over a period of several weeks involving wild avian species in their natural environment pursing their natural food sources and at pre-existing nesting sites located at the top of cell towers.

Test \#1: A final open air trout rearing pond at the Milford Fish Hatchery in Milford, NH (longitude and latitude coordinates: 42.8515, -71.6894) experiences $\sim 30 \%$ annual predation loss from Osprey (OSPR). The final rearing pond is approximately $20 \times 50 \mathrm{~m}$ which is surrounded by a mix of $20-30 \mathrm{~m}$ tall hardwood and pine trees from which the Osprey would frequently perch at the edge of the tree line on the southerly or westerly side. Foraging attempts were initiated from the tree perch or from fly-by attacks from an altitude of $\sim 80 \mathrm{~m}$. The tree line surrounding the east, south and west sides of the holding pond began at a distance of $80 \mathrm{~m}$ from the pond edge.

The resident Osprey behavior was observed from a location that was $\sim 50 \mathrm{~m}$ north of the pond between 
sunrise and sunset on eight days without lights (control data) and on five additional days with lights (test data) during the month of late September to early October 2014. A single light array was positioned at a corner of the pond for the first day and midway along one side of the pond for the remaining four days of testing. The authors documented site of initiation, type, and outcome of all foraging attempts observed. Unsuccessful attempts included low passes over the pond and dives that did not produce fish. The Osprey left the fishery area and may have started their winter migration at the conclusion of the test.

Test \#2: A LED device was installed at the top of cell towers in close proximity to nests during the Osprey nesting season of 2015 (April-August 2015). The cell towers are located throughout the central region of Maine located throughout the mid-coast and mid-highland regions of Maine (longitude and latitude coordinates: $\quad 44.8279-68.9144, \quad 44.8441-68.8299$, 44.0756-69.0887, 44.9901-69.0146, 43.9703-69.5139, 44.9016-68.7824). All cell towers were known to have been active Osprey nesting sites in the previous year. The pre-existing nest material was not removed from the towers. The cell towers consisted of multiple layers of $\mathrm{Tx} / \mathrm{Rx}$ antennas mounted on a three-sided lattice design with the maximum height between 70-100 m AGL. The first three sites were test sites and the last three sites were used as control sites in which non-functioning dummy devices were installed in close proximity to the nests. The Osprey had occupied all of the nests and laid eggs in five of the six nests located on the cell towers at the time that the devices were installed. The Animal and Plant Health Inspection Service (APHIS, an agency of the United States Department of Agriculture) removed the eggs from two of the three test sites (Tower \#1 and \#2) during the installation of the devices. The three test towers had deterrence devices installed that contained a built-in camera which recorded video whenever motion was present. All devices were installed 1-2' higher than the nest with the LED light projecting across and into the Osprey nest. Motion in the vicinity of the nest activated the flashing LED and played a scary sound of an Osprey being attacked by an Eagle. The LED light flashed for 10 seconds at a variable rate between $1-3 \mathrm{~Hz}$ concurrently. On two of the towers, a 10-12 second audio recording of an Osprey being attacked by a Bald Eagle was played concurrently when the LED lights were flashing. The devices were programmed to play once after 20 mins if no motion was detected. The sites were monitored every few weeks and the motion triggered video recordings downloaded.

\subsection{Experimental Materials}

The LEDs consisted of a monochromatic LED manufactured by Epiled capable of emitting a Gaussian beam of light with a peak emission of 395 $\mathrm{nm} \pm 15 \mathrm{~nm}$ which can be electronically controlled to pulse with 0.001 second accuracy were fabricated into outdoor devices consisting of multiples of $100 \mathrm{~W}$ LEDs. The wall plug power conversion efficiency stated by the LED manufacturer was $12 \%$.

Test Site \#1: The monochromatic LED devices capable of emitting a Gaussian beam were focused to create an illumination beam of light (FWHM \pm 30 degrees) directed to airspace of the normal flight path used by Osprey. The LED light beam was directed across the fish pond at an angle of approximately 20degrees above horizontal. Nine $100 \mathrm{~W}$ LEDs were pulsed at rate of $1.5 \mathrm{~Hz}$ with ON/OFF time of 250 $\mathrm{ms} / 500 \mathrm{~ms}$ producing a calculated peak intensity of $1.410^{-6} \mathrm{~W} / \mathrm{cm}^{2}$ at $100 \mathrm{~m}$ distance.

Test Site \#2: A single $100 \mathrm{~W}$ device, a $3 \mathrm{~W}$ speaker, and camera with motion detection software were mounted on cell towers. The LED was pulsed at rate of $2 \mathrm{~Hz}$ with ON/OFF time of $250 \mathrm{~ms} / 250 \mathrm{~ms}$ producing a calculated peak intensity of $4.210^{-5}$ $\mathrm{W} / \mathrm{cm}^{2}$ at $3 \mathrm{~m}$ distance.

\subsection{Experimental Set Up}

Animal care and experimental procedures were in 
accordance with the legal requirements of USA. No additional license was required for this study. FWS and APHIS (Animal and Plant Health Inspection Service) personnel were involved in the setup of the experiments.

\section{Results and Discussion}

\subsection{Results}

Test \#1: OSPR foraging success was significantly higher in the absence of lights $\left(x^{2}=8.062, \mathrm{df}=1, \mathrm{p}=\right.$ 0.005).

OSPR proportions of dives (successful and unsuccessful combined) and passes (never successful) were not significantly different in the presence and absence of lights $\left(x^{2}=0.441, \mathrm{df}=1, \mathrm{p}=0.507\right)$.

All species exhibit caution when approaching the site while the monochromatic LEDs were operating. The avoidance behavioral response increased as the distance to the mono-color LEDs decreased. The behavioral response to the mono-colored light, ranged from subtle changes of flight direction or altitude to dramatic responses which included complete reversal of flight direction at closer distances where the intensity of light was greater. A difference in the behavioral response to UV light and time taken to initiate a foraging attempt by Osprey at the fish hatchery was noted but data were not recorded.

Test \#2: Behavioral response to UV light by Osprey nesting in all cell towers with functional devices could be characterized as being significantly annoyed by the devices. The Osprey on Tower \#1 showed the most dramatic change in behavior from long term exposure to a working device. The Osprey started to build a second nest at the edge of the illumination pattern of the device within a few days. The first and second nests were abandoned when they built a third nest even farther away from the device during the third and fourth week. The construction of the fourth nesting site located directly behind the device was underway during the seventh and eighth week which coincided with the Osprey regularly defecating on the device. By
Table 1 Number of successful and unsuccessful Osprey foraging attempts.

\begin{tabular}{llll}
\hline OSPR & No lights & Lights & Total \\
\hline Successful & 24 & 6 & 30 \\
Unsuccessful & 25 & 27 & 52 \\
Total & 49 & 33 & 82 \\
\hline
\end{tabular}

Table 2 Number of Osprey foraging attempts.

\begin{tabular}{llll}
\hline OSPR & No lights & Lights & Total \\
\hline Dive & 36 & 22 & 58 \\
Pass & 13 & 11 & 24 \\
Total & 49 & 33 & 82 \\
\hline
\end{tabular}

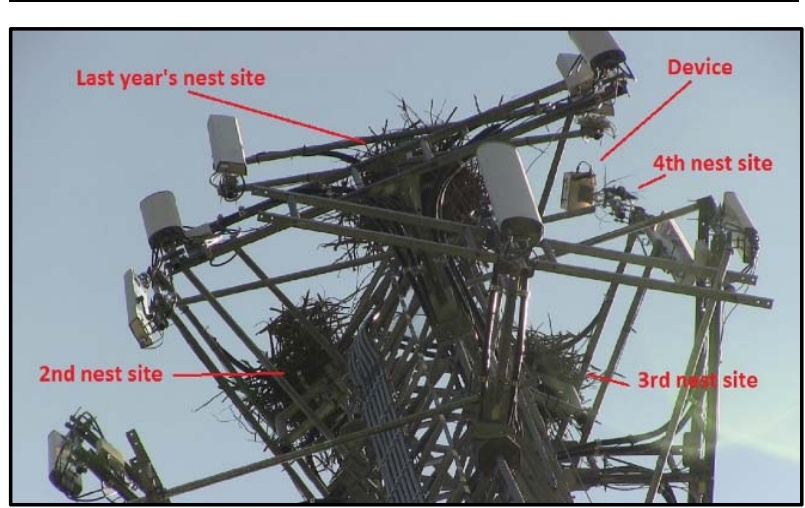

Fig. 1 The osprey at tower \#1 quickly built alternative nests in the cell tower to move farther away from device. The daily visits decreased rapidly. The Osprey never stayed on the nest at night after the first night.

the end of the ninth week, all of the nests in the cell tower were abandoned.

The sound unit on Tower \#2 malfunctioned soon after being installed. The avoidance behavior to the LED light was observed. It is believe that the scary sounds are complementarity to the behavioral response induced by the LED, but further study would be required to quantify any benefit. No change in the nesting and chick rearing behavior of the Osprey were noted due to the presence of non-functional devices located on the control cell tower.

The device on Tower \#1 and \#3 continued to operate throughout the next several months. The daily number of in-nest visits at the tower, excluding fly-by events, rapidly decreased from the initiation of the test. The number in-nest visits at Tower 2 is included in the data up to the date when an egg was laid and the Osprey remained on the nest continuously. The 
Osprey from Tower \#1 left the tower during the 9th week. A portion of the video recordings were lost when the hard drives storage capacity was exceeded.

Once the Osprey claim nest site in the cell tower test, their behavior included extreme actions (fighting, adapting, relocating the nest, and learning avoidance behaviors) to remain at the tower. The authors hypothesize that the Osprey are strongly driven by hormonal signals since this activity involves mating, nesting and rearing young. Hormonal effects are difficult to overcome.

The changes observed in the Osprey behavior during the time the device was used includes:

- Display of extreme agitation to the stimulus of the devices;

- Complete avoidance of the nest site at night unless they are brooding in a nest;

- Decreasing frequency of visits to the nest resulting in abandonment of the nest;

- Minimization of motion while occupying the nest;

- Altered flight path approach to the tower limiting triggering of the motion detection feature of the devise;

- Building alternative nest sites at locations on the tower to minimize triggering the device;

- Modification of the nest structure (increase wall height) which minimized exposure to the light while in the nest;

- Ever increasing frequency of defecation on the device.

No noticeable change of the Osprey nesting and chick rearing activities were noted in the control roup due to the installation of the non-functional devices.

\subsection{Discussion}

Measuring behavioral influence of non-lethal stimulus of light and sound with wild Osprey is a real-life experiment with many uncontrolled variables. Foraging for food and the raising of young are behavioral

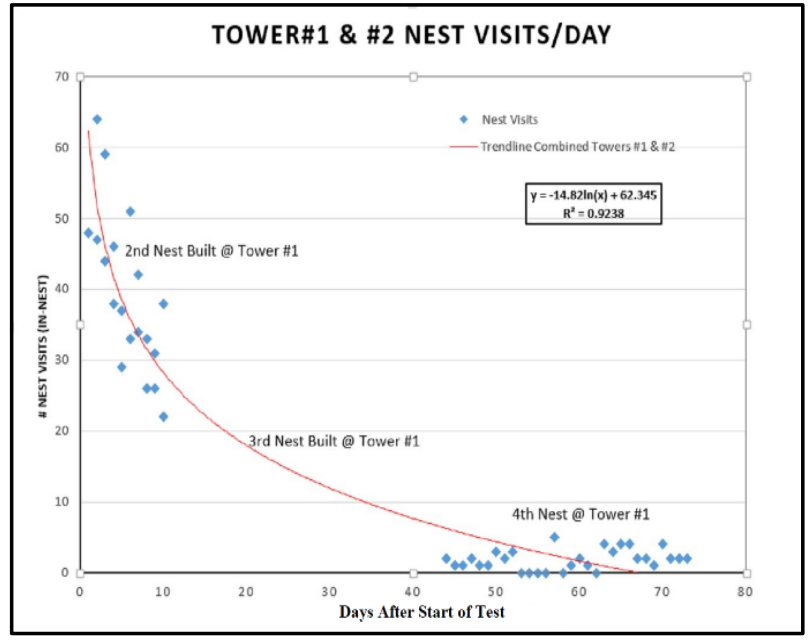

Fig. 2 The number of visits/day to the nest at Tower \#1 and \#2 rapidly decreased.

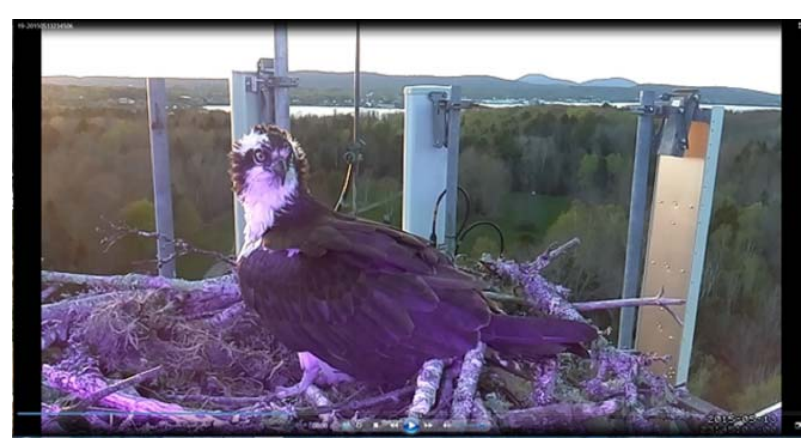

Fig. 3 Annoyed Osprey responding to the device.

activities that highly motivate all species which represents a high threshold of measurement. The change in the success rate of foraging attempts indicates a significant interference with the near instantaneous interference with their ability of visual perception. The influencing upon their nesting behaviors confirms that the non-lethal stimulus can be a long term annoyance. It also indicates that highly motivated cognitive thoughts can overcome the instantaneous influence of the stimulus which implies the potential inherent risk to long term habituation.

Understanding the neurophysiological mechanisms of the instantaneous augmented behavioral response enables optimizing the conditions under which it may be used. This requires a discussion of the morphophysiological organization of the visual systems which is dependent upon the unique characteristics of the eye, the post-receptoral mechanisms of the neuro 
pathways, and the neuro-motor mechanisms. The initiation of vision starts with incidence of light upon photoreceptors. Photons striking a photoreceptor must be of sufficient intensity and appropriate energy to cause a photochemical reaction. The photochemical reaction of the photoreceptor creates a nerve impulse that is transmitted through an arrangement of retinal neuro transmitters that enables signal pre-processing, such as center-surround and support color-opponent processing of a receptor field. The middle layer of the retina consists of horizontal cells, bipolar cells and amacrine cell is where the differential neuro signal process from the surrounding cones occurs. All signals originating in the photoreceptors and arriving at the ganglion cells must pass through the bipolar cells to the ganglia cell. This means that bipolar cells are a part of both the direct and indirect paths while the horizontal cells and amarcine cells are a part of the indirect path. The neuro impulses that the ganglia cells send to the brain has little to do with the absolute intensity of light shining on the retina, but signals the result of a comparison of the amount of light hitting a certain spot on the retina with the average amount falling on the immediate surround. The ganglia cells are connected to the second cranial nerve (optic nerve) transmits visual information to the vision centers of the brain. The optic nerve is generally larger than the spinal nerve which indicates the importance of vision to avian species. Numerous neurophysiological responses involve visual perceptions from the sensory inputs from the eye occur within the brain which leads to behavioral responses.

Humans and monkeys are capable of accommodating more than 100:1 ratio of light contrast, while avian species are capable of accommodating less than 11:1 ratio light contrast [14]. Avian species are commonly recognized to have better visual acuity and lower dynamic range compared to humans.

High brightness, monochromatic LEDs are capable of inducing a saturated neuro impulse on a single photoreceptor channel (cone color) causes disruption to a light adapted eye. The light adapted eye attempts to adapt by a pupil dilation response which reduces the overall intensity of illumination striking the photoreceptors. The change of the pupil size modifies the set point of the contrast sensitivity function that the eye accommodates. The repetition of additional series of brief pulses that are appropriately sequenced in time induces the vision system to attempt to adapt. A condition can be established where the eye is constantly ajusting to a changing set of light conditions. Exposure to a series of brief pulses of light consisting of wavelengths matching the peak absorption wavelength of the short wavelength photoreceptors of the specie have been found to efficiently cause this condition. The CSF threshold intensity necessary to produce this effect is defined by the brightness of the illuminated region of the image of the light adapted

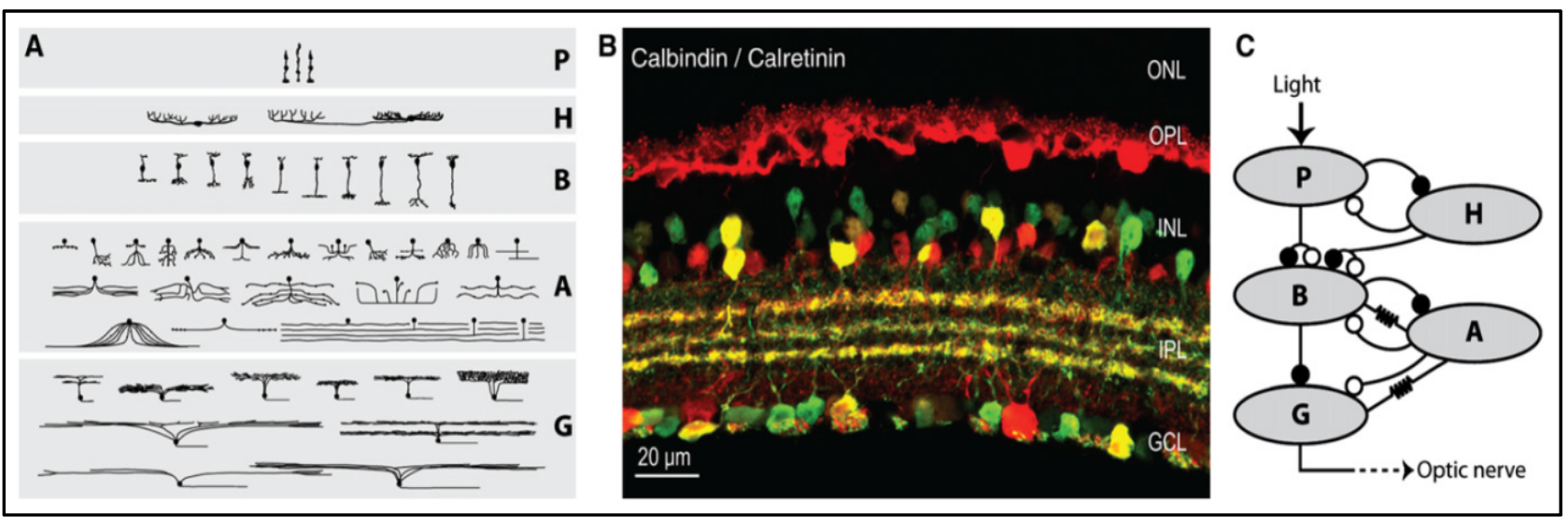

Fig. 4 Adapted from Ref. [11]. Retinal circuitry retinal circuitry (A) Diversity of retinal cell types. For all five classes of retinal neurons-photoreceptors (P), horizontal cells (H), bipolar cells (B), amacrine cells (A), and ganglion cells (G); (B) Specificity of retinal wiring; (C) Schematic drawing of connections between the basic cell classes. 


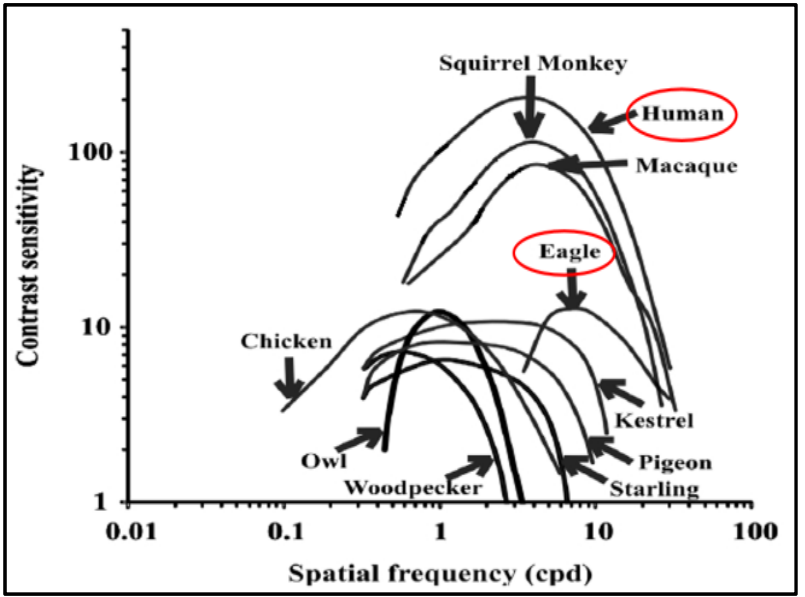

Fig. 5 CSFs (Contrast sensitivity functions) comparison between primates and avian species. Adapted image [14].

eye, plus the intensity required to exceed the CSF ratio. When the photoreceptor regions illuminated by the monochromatic LEDs is overwhelmed in this manner, the ability of the species to maintain a balanced optical flow is defeated. Visual perception of the affected photoreceptor regions nterfere with the post-receptoral mechanisms of the neuro pathways leading to behavioral responses. The authors call this condition an augmented behavioral response.

Avian morphological structure may differ when compared to other animals, but the neuro processing pathways and functions of the tectofugal and thalamafugal pathways are similar across the animal kingdom. Most primates' visual input is processed in the lemnothalamic, or thalamofugal, pathway whereas the tectofugal is the major pathway in birds [15]. The tectofugal pathway is considered to be the center of auditory and visual motion and has a direct connection to forebrain functions [12]. The thalamofugal pathway connects the left and right hemispheres of the brain and involves visual intensity and pattern discrimination. There are many different functions of the forebrain, including processing of cognitive, auditory, sensory, and visual information. The perception of visual information is necessary for the brain to initiate behavioral changes. As a reference, humans are believed to have visual process areas that are sub-divided into 32 distinct areas with over 300 interconnections.
The avian brain Midbrain is a portion of the central nervous system associated with vision, hearing, motor control, sleep/wake, arousal (alertness), and temperature regulation. The Cerebrum is responsible for the integration of complex sensory and neural functions and the initiation and coordination of voluntary activity in the body. The Tectofugal pathway (extrastraite visual pathway) is a common visual pathway connecting the optic tectum with the forebrain. It is involved in tasks such as orienting and attention, and other tasks that benefit from integrating information across senses. The multiple functional area, is sensitive to motion and is used in the perception of shapes. The Thalamofugal pathway is a set of central visual pathways in birds that is equivalent to the mammalian geniculocortical pathway that processes "where" (dorsal) and "what" (ventral) in the telencephalon. The Rt (nucleus rotundus) is a major relay station in the tectofugal pathway of the avian visual system. Color discrimination activities produced strong and lateralized activation in the Rt while motion detection and ground discrimination are supported by other brain centers. The Gld (lateral geniculate nucleus, dorsal part) is a relay center in the thalamus for the visual pathway consisting of alternating layers of neurons (grey matter) with optic fibers (white matter). Entopallium is the major thalamorecipient zone, within the telencephalon, of the tectofugal visual system. Mesopallium has been shown to involve learning and long term memory functions which are believed to be associated with reward seeking behavior. The Hyper pallium is the telencephalic target of the thalamofugal visual pathway of birds. The telencephalic is located in a portion of the cerebrum consisting of two cerebral hemispheres plus a small mid-line component called lamina terminalis which is the center sensory impulses; motor functions; planning and organization; sense of smell; sense of touch; determines intelligence; determines personality, and interpretation sensory impulses. 
Chromatic contrast and edge detection is believed to play an important role in visual perception by enabling the identification of objects and tracking motion. An example of a human color balance (eye adapted) image and simulated chromatic contrast and edge detection signals are illustrated in Fig. 6. The identical opponent colors (B-G) and edge detection technique is illustrated in Figs. 6 and 7 except that an increase brightness in the blue color channel due to being illuminated with a blue colored LED is simulated. Note the dramatic degradation of the signals strength during neuro-processing. The human neuro impulse signals are illustrated using the color opponent models (simple chromatic contrast) and center-surround (edge detection) provide insight to non-human systems.

An augmented behavioral response occurs when the light adjusted contrast sensitivity function for the species is exceeded. This involves the neurophysiological blocking mechanism of the visual system inducing temporary disruption of vision within the region of the field of view. Humans commonly experience temporary loss of vision by a bright flash of light in the eye of any color of light. The most effective color of light to induce the augmented response involves the short wavelength cone. An intensity striking the eye of avian species greater than $10^{-6} \mathrm{~W} / \mathrm{cm}^{2}$ or $210^{6}$ photons $/ \mathrm{mm}^{2}$ which are matched to the wavelength of the short wavelength photoreceptor initiates the augmented behavioral responses. A light adapted eye that accommodates a brightly lit background will require a corresponding increase in intensity from the LED to compensate. The augmented response leads to significantly altered behaviors of the species tested. Pupillary constriction and dilation of the light adapted eye are induced by exposure to a series of brief pulses of light consisting of wavelengths matching the peak absorption avelength of the short wavelength photoreceptors of the specie. The pulsing interval of the mono-colored LED can be selected to keep the

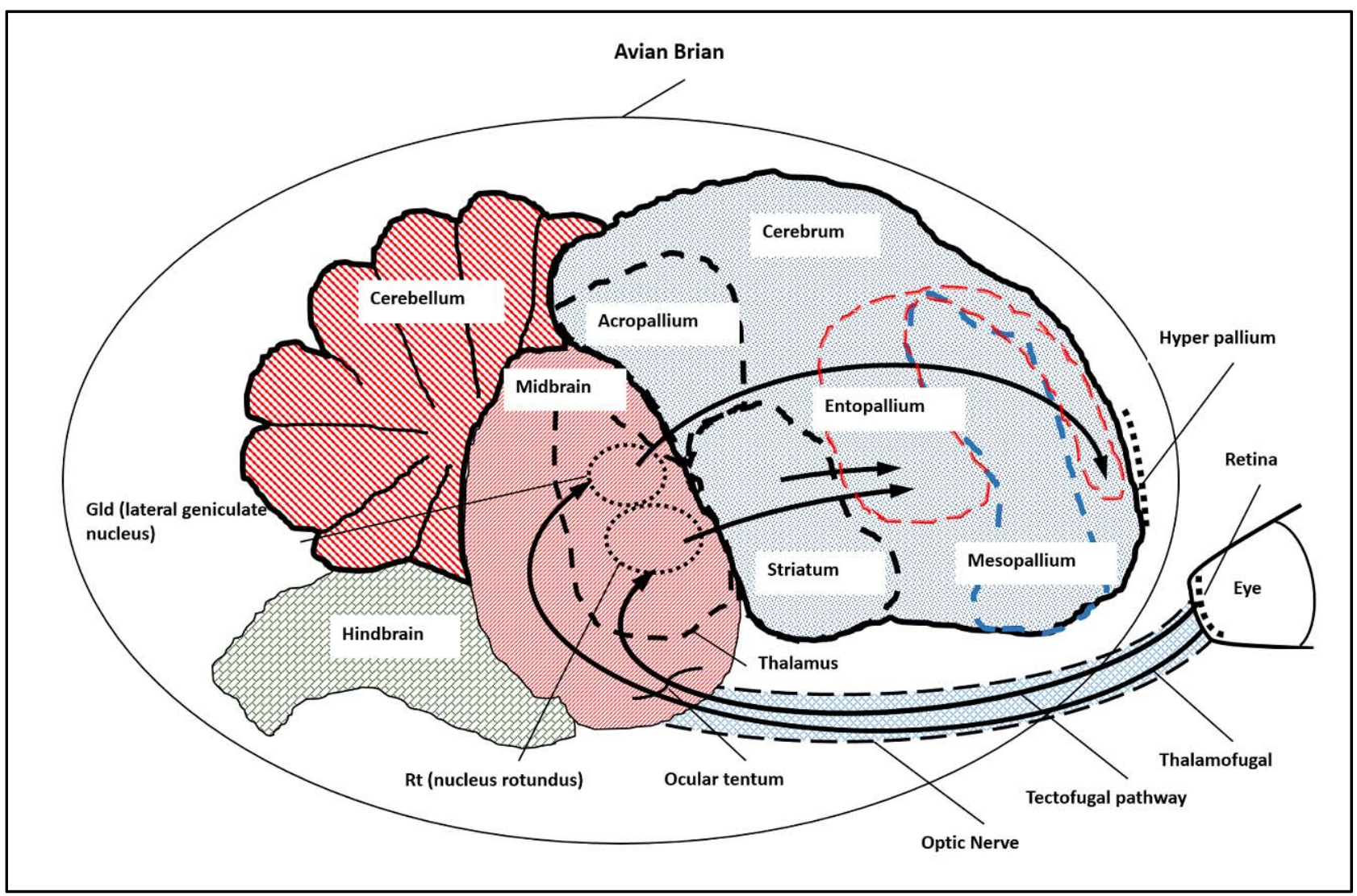

Fig. 7 The avian brain (parasagittal section: dorsal—up, anterior-right. 


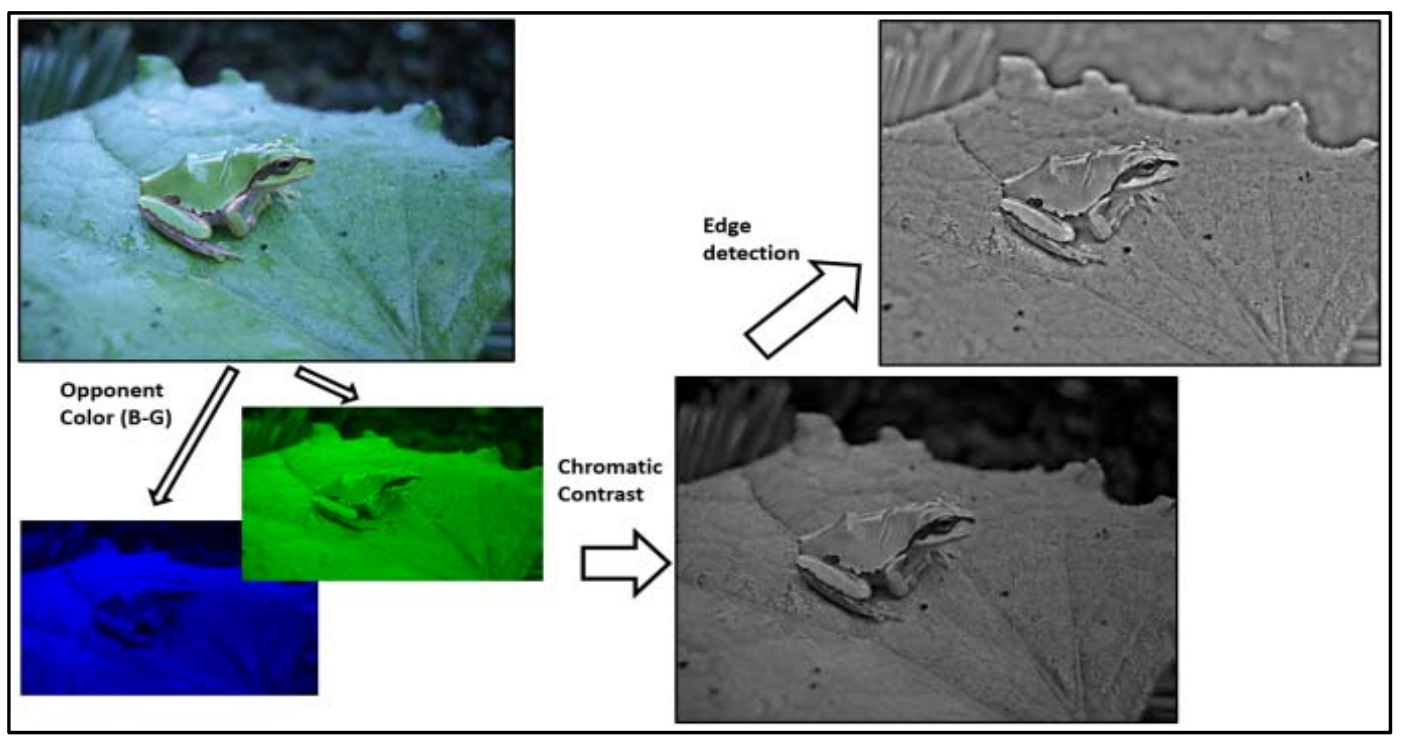

Fig. 6 A color image of a green frog on a green leaf consists of 3 primary human colors (R, G, B). The opponent colors (B-G) is an illustration of chromatic contrast and edge detection which compares surrounding pixel values to enhance the localized contrast of the photoreceptor region.

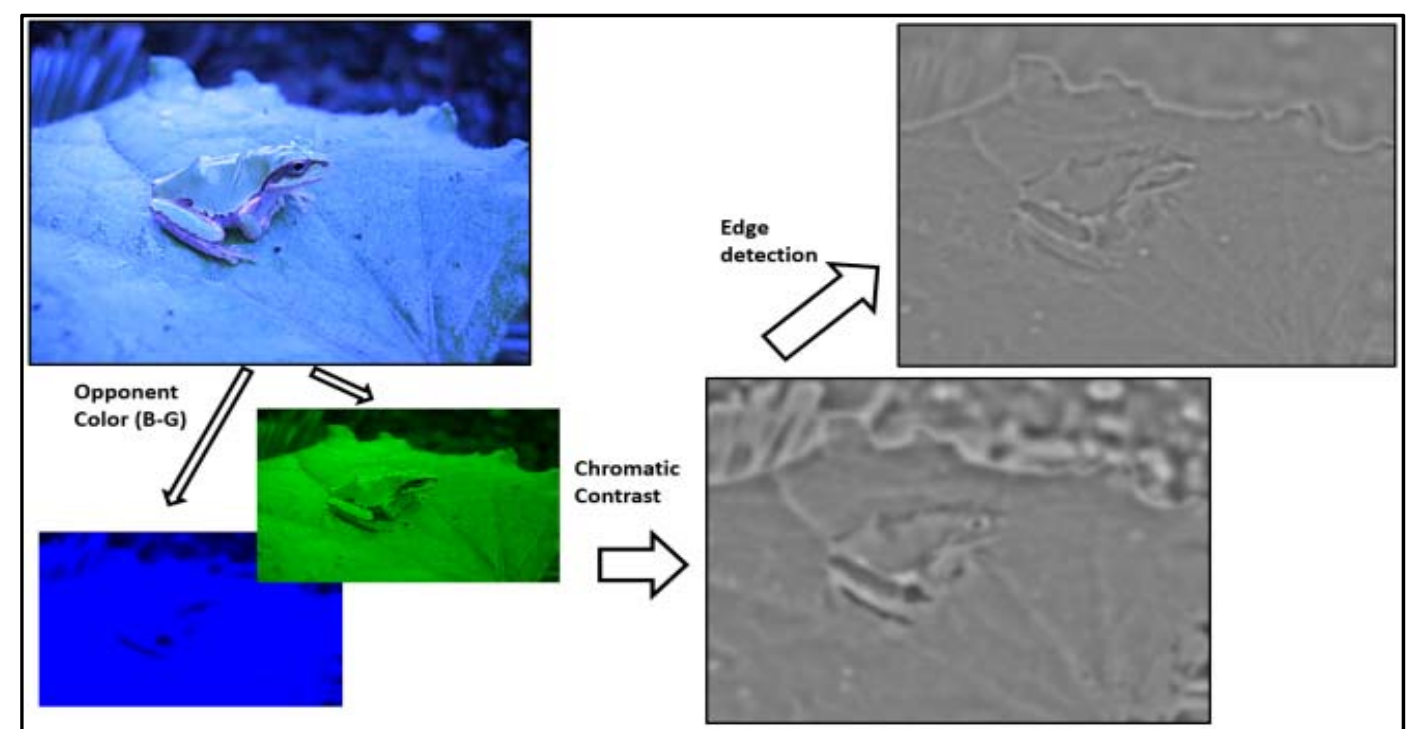

Fig. 7 The blue channel brightness of the image has been increased to simulate the saturated short wavelength cone channel from increased illumination of a blue colored LED.

pupil of the eye in a constant unstable state thereby preventing the specie from adapting to the pulsing LED light source and acquiring visual information. When the oculo-neuro network is overwhelmed in this manner, the ability of the species to maintain visual perception is effectively defeated enabling a new technology for non-lethal wildlife deterrence.

\section{Conclusions}

LEDs that are color matched to the short-wavelength cones of avian species interfere with the ability of the oculo-neuro-motor network and result in an altered behavioral response to otherwise predictable species behaviors. An augmented behavioral response occurs when the light adjusted CSF (contrast sensitivity function) for the species is exceeded. This involves the neurophysiological blocking mechanism of the visual system inducing temporary disruption of vision within the region of the field of view. When the oculo-neuro network is overwhelmed in this manner, 
the ability of the species to maintain visual perception is temporarily defeated or severely degraded. The results of an overwhelmed neurological condition result in the interference of neurophysiological processes of the vision system and brain controlling edge detection, motion, optical flow, afterimage, illusions, and flicker fusion. The loss of visual perception of the region of the field of view will be noticed by the species. The behavioral response to the sudden and repeated loss or degradation of vision may be either voluntary or involuntary. The animal species is unlikely to consciously choose to remain in this environment but likely to perceive the light source as either a threat or a significant source of discomfort and move away.

The recent development of high brightness monochromatic LEDs enable the unique ability to stimulate individual color channels of the vision system. The excessive stimulation of a color channel, exceeding the contrast sensitivity ratio of the light adapted eye, will interfere with the neurophysiological visual perception processes of the eye and brain resulting in a change in behavioral responses. This novel technique will enable a unique way to study the interactions of numerous neurophysiologic processes and can be utilized as a non-lethal deterrence to effect avian behavior to mitigate avian-human conflict in areas such as airports, airplane bird strikes, and wind turbines while not being seen by humans.

\section{Acknowledgment}

This work was supported by grant No. IIP-1350562 from the National Science Foundation. NH Audubon Society provided assistance in tabulating the field results. Support at the test sites was provided by US Cellular, and NH Fish and Game Department-Milford Fish Hatchery.

\section{References}

[1] Jacobs, G. H. 2015. "Evolution of Colour Vision in
Mammals.” Philosophical Transactions of the Royal Society B: Biological Sciences 364 (1531): 2957-67.

[2] Collin, S. P., Davies, W. L., Hart, N. S., and Hunt, D. M. 2009. "The Evolution of Early Vertebrate Photoreceptors." Philos. Trans. R. Soc. Lond. B. Biol. Sci. 364 (1531): 2925-40.

[3] Hart, N. S. 2001. "The Visual Ecology of Avian Photoreceptors.” Prog. Retin. Eye Res. 20: 675-703.

[4] Hart, N. S., and Hunt, D. M. 2007. "Avian Visual Pigments: Characteristics, Spectral Tuning, and Evolution.” 169 (1): S7-26.

[5] Bennett, A. T. D., and Cuthill, I. C. 1994. "Ultraviolet Vision in Birds: What is Its Function?” Vision Res. 34: 1471-8.

[6] Håstad, O., Victorsson, J., and Ödeen, A. 2005. "Differences in Colour Vision Make Passerines Less Conspicuous in the Eyes of Their Predators." Proc. Natl. Acad. Sci. 102: 6391-4.

[7] Honkavaara, J., Siitari, H., and Viitala, J. 2004. "Fruit Color Preferences of Redwings (Turdus Iliacus): Experiments with Hand-raised Juveniles and Wild-caught adults.” Ethology 110: 445-57.

[8] Osorio, D., and Vorobyev, M. 2008. "A Review of the Evolution of Animal Colour Vision and Visual Communication Signals.” Vision Res. 48: 2042-51.

[9] Rubene, D., Håstad, O., Tauson, R., Wall, H., and Ödeen, A. 2010. "The Presence of UV Wavelengths Improves the Temporal Resolution of the Avian Visual System.” $J$. Exp. Biol. 213: 3357-63.

[10] Gollisch, T., and Meister, M. 2009. "Eye Smarter than Scientists Believed: Neural Computations in Circuits of the Retina.” Neuron 65 (2): 150-64.

[11] Marín, G., Salas, C., Sentis, E., Rojas, X., Letelier, J. C., and Mpodozis, J. 2007. “A Cholinergic Gating Mechanism Controlled by Competitive Interactions in the Optic Tectum of the Pigeon.” J. Neurosci 27: 8112-21.

[12] Odeen, A., and Hastad, O. 2003. "Complex Distribution of Avian Color Vision Systems Revealed by Sequencing the SWS1 Opsin from Total DNA.” Mol. Biol. Evol. 20 (6): 855-61.

[13] Givago, da S. S., Gomes, B. D., and Silveira, L. C. L. 2011. "Comparative Neurophysiology of Spatial Luminance Contrast Sensitivity.” Psychology \& Neuroscience 4 (1) 29-48.

[14] Engelage, J., and Bischof, H. J. 1993. "Vision, Brain, and Behavior in Birds: The Organization of the Tectofugal Pathway in Birds: A Comparative View." MIT, Cambridge, MA. 